Pensamiento Crítico N. ${ }^{\circ}$ 9, pp. 77-98

\title{
Efectos de la congestión de las redes de transmisión en la competencia en mercados eléctricos de producción hidrotérmica
}

Mg. Jaime E. Luyo

\section{RESUMEN}

En este ensayo se analizan los efectos de la congestión de las líneas de transmisión de una red interconectada en la competencia en un mercado spot mayorista de generación, con producción hidro-térmica de electricidad, en un ambiente oligopólico. Se desarrolla un modelo de equilibrio dentro del enfoque Nash-Cournot.

Palabras clave: Poder de mercado, congestión, mercado spot, equilibrio NashCournot, withholding.

\begin{abstract}
This essay analyzes the network-transmission lines congestion effects on the wholesale generation spot market competence, with a hydrothermal electricity production, and within an oligopolic environment. It develops a Nash-Cournot equilibrium model.
\end{abstract}

Keywords: Market power, congestion, spot market, Nash-Cournot, equilibrium, withholding. 


\section{Jaime €. Luyo}

\section{INTRODUCCIÓN}

La experiencia internacional de desregulación del sector eléctrico hacia el mejoramiento de bienestar social, indica que este proceso se sustenta en la competitividad de los mercados eléctricos mayoristas; reconociéndose que uno de los obstáculos para alcanzar este objetivo es el poder de mercado $(\mathrm{PM})^{1}$, que se define como la capacidad de alterar con beneficios, los precios fuera de los niveles competitivos ${ }^{2}$, y que ejerce un generador cuando reduce su producción o eleva el precio mínimo a que está dispuesto a vender su producción para cambiar el precio de mercado ${ }^{3}$. Además, las empresas de generación que compiten entre ellas, dependen de la capacidad del sistema de transmisión; y cuando este tiene congestión, el mercado se fragmenta en zonas cuyos mercados pueden ser más concentrados donde el generador dominante puede ejercer PM local ${ }^{4}$, produciéndose pérdidas de eficiencia debidas a ambos factores ${ }^{5}$. Dadas las características especiales de la electricidad, entre otras, la congestión en la transmisión es también un impedimento para tener un mercado competitivo ${ }^{6}$, la generación, transmisión y distribución están regidas vinculadas a las leyes físicas de Kirchoff ${ }^{7}$, la demanda y oferta se deben satisfacer todo el tiempo por seguridad del sistema eléctrico, y son dependientes del clima y meteorología, particularmente la producción hidroeléctrica; lo que hace inevitable que se produzca, aun transitoriamente, el ejercicio de poder de mercado y congestión en algunos sectores de la red de transmisión que conecta a productores y consumidores, por lo que se tienen que adoptar medidas de mitigación del PM de los generadores dominantes en el mercado mayorista por los entes reguladores.

Para que el mercado eléctrico al por mayor sea competitivo, se requiere que generadores y consumidores tengan libre acceso a las líneas de transmisión, ya que este

1 Joskow, 2003.

2 Stoft, 2002.

3 Borenstein, 2000.

4 Twoney, P. et al., 2005.

5 Cho, In-Koo y Hyunsook Kim, 2002.

6 Ferc, 2003; Leisutre y Eto (2003).

7 Kirchhoff, Leyes de: leyes físicas que gobiernan el voltaje (voltios) y corriente (amperio) en las redes eléctricas, y los flujos de potencia (vatios) eléctrica.

- "en todo nodo la suma algebraica de las corrientes debe ser cero" (ley de los nodos; equivalente al equilibrio oferta-demanda en el mercado eléctrico).

- "en toda malla (loop) la suma algebraica de las caídas (diferencia) de potencial eléctrico debe ser cero" (ley de mallas; equivalente a las pérdidas de capacidad al transportar la electricidad de un nodo a otro). Ver Schweppe et al. (1988), pp. 32, 152-153. 


\section{Efectos de la congestión de redes de transmisión en mercados eléctricos}

medio vincula a los agentes y transporta la electricidad. La electricidad respecto de otros bienes o servicios tiene características diferenciadas, entre otras, como: el simultáneo balance oferta-demanda en todos los puntos o nodos (barras) de la red eléctrica todo el tiempo; la imposibilidad de almacenamiento económico; la no-controlabilidad del flujo de electricidad en la red debido a la variación permanente de la demanda, la entrada o salida de generadores y consumidores, y otras contingencias. Estas características producen inevitables externalidades ${ }^{8}$, como en el caso de la congestión ${ }^{9}$ de alguna línea de transmisión.

En el Perú, desde el año 2005, el Comité de Operación Económica del Sistema Interconectado Nacional (COES-SINAC) está reportando problemas de congestión periódica en líneas de transmisión críticas para el abastecimiento de electricidad con eficiencia económica y seguridad, para las diferentes regiones del país, lo que al haberse agudizado en el año 2007 (ver Figura 1 de Anexo), debido a la congestión de la línea de transmisión (Lima - Chimbote $220 \mathrm{Kv}$ ) que une eléctricamente el centro con el norte del país, produciéndose la elevación desmesurada de los costos marginales de la zona norte con relación al centro y sur, como puede observarse en el gráfico mostrado. Como respuesta, el gobierno tuvo que emitir el 25-11-2007 el Decreto de Urgencia N. ${ }^{\circ} 0446$ 2007, que establece que por consideraciones de congestión de instalaciones de transmisión, el COES deba despachar unidades (de generación) fuera del orden de mérito de costos variables, tomando en cuenta los criterios de optimización en la operación del Sistema Eléctrico Interconectado Nacional (SEIN), los costos variables de dichas unidades no serán consideradas para la determinación de los costos marginales del SEIN. Esta situación actual de insuficiencia de capacidad de transmisión y generación eléctrica, refleja la ausencia de una adecuada planificación del desarrollo eléctrico y que las medidas que se han ido adoptando tienen un carácter más coyuntural que estratégico (como la ineficacia de la llamada Ley de Generación Eficiente).

Desde nuestro particular punto de vista, también observamos que no existe un marco analítico que sustente objetiva y técnicamente las políticas y medidas que se vienen adoptando desde la crisis del 2004, en el sector eléctrico, y en este artículo pre-

8 Luyo, J.E. "La Reforma del Sector Eléctrico en Perú: La crisis del 2004 y cambios necesarios". En: Revista de la Facultad de Ciencias Económicas, UNMSM, Año X, N. ${ }^{\circ}$ 30, Lima, julio-setiembre 2006.

9 Congestión: Límite de capacidad de transmisión de sus líneas por efectos térmicos, salida de un componente, caída de voltaje, o por límite de estabilidad y contingencias. 


\section{Jaime E. Luyo}

sentamos un modelo matemático que permite analizar los efectos de la congestión en las líneas de transmisión de electricidad en la competencia, para mercados eléctricos con generación hidrotérmica en un ambiente oligopólico.

\section{REVISIÓN DE LA LITERATURA}

Según Borenstein et al. (2000), usando un modelo de Cournot, estudian el caso para una sola línea que conecta un generador y un consumidor localizados en cada nodo terminal de la línea; concluye que la capacidad de transmisión determinará el grado de competencia entre generadores, y este será el centro de los problemas de poder de mercado en un mercado desregulado; y la capacidad de las líneas de transmisión, determinará el grado en que compiten generadores en diferentes localizaciones. Stoft (1997) señala que una línea congestionada separa el mercado en dos regiones no competitivas, posibilitando el ejercicio de poder de mercado por los generadores de cada región, y que un generador puede reducir su producción congestionando una línea, para así incrementar su poder de mercado, y que puede producirse el equilibrio de un duopolio, sin producirse ningún flujo de potencia en la línea; Hogan (1997) llama la atención sobre el alcance de las restricciones de la red de transmisión que determina el grado de poder de mercado local, observando que, según la topología de la red la congestión en esta puede ser en ciertas circunstancias; mayor aún en períodos fuera de pico (fuera de la máxima demanda); y que al considerar las complejas interacciones en la transmisión, la topología del mercado está determinada por la distancia eléctrica y no la distancia geográfica. Esto último ha sido previamente estudiado por Wu et al. (1995), esclareciendo, además, que es incorrecta la analogía entre transmisión de electricidad y transporte de bienes, ya que la diferencia de precios entre los nodos (barras) de una línea de transmisión de potencia no representa el costo de transporte de esta (o el precio de congestión) y solo en el caso de la línea congestionada; y que la potencia también puede fluir -en algunos casos- de un nodo de mayor precio a otro de menor precio, todo esto no sucede en el mercado de bienes comunes.

Green (1998) estudia el sistema de transmisión de Inglaterra y Gales incluyendo las pérdidas de transmisión y congestión, concluyendo que el método de precios spot nodales óptimos es preferible al sistema de precios uniformes; ya que proporciona mejores señales económicas cuyo beneficio mayor es el de incentivar las inversiones. Harvey y Hogan (2000) concluyen que el modelo de precios nodales para el mercado eléctrico, 


\section{Efectos de la congestión de redes de transmisión en mercados eléctricos}

por razones de eficiencia y para mitigación del poder de mercado local cuando hay congestión en la red, es superior al modelo de precios zonales que, más bien, incrementa los beneficios del generador local dominante. Wolak (2003a) dice que en el anterior régimen de monopolio vertical integrado, las diferencias de precios de energía al por mayor a través de las regiones se debían a las diferencias de los costos de producción locales para el monopolista; pero, en el actual régimen de mercado al por mayor, la extensión del poder de mercado que pueden ejercer las empresas en cada lugar de la red, puede causar mucha mayor diferencia en el precio de la electricidad entre las regiones con relación a la diferencia de los costos variables de las unidades de generación más caras en estas, debido a la congestión en la transmisión. Considerando que los países de Latinoamérica tienen problemas de una transmisión insuficiente y congestionada, con generación hidroeléctrica predominante, con potenciales problemas de poder de mercado local y sistémico, recomienda para el diseño de mercado adoptar la estrategia de despacho basada en costos, a cargo de un operador independiente $\left(\mathrm{ISO}^{10}\right.$, preferentemente de propiedad estatal), que además opere la transmisión, facilitando, así, la competencia en el mercado mayorista. Ehrenmann y Neuhoff (2004) reportan el estudio de la red integrada de Nueva Zelanda-Bélgica-Alemania-Francia, concluyendo que en el diseño de mercado para la comercialización de la electricidad, en una red eléctrica con restricciones en la transmisión, la opción de diseño poo ${ }^{11}$ de precios spot nodales (que integra los mercados de transmisión y de energía, con un operador central del sistema) reduce la posibilidad de ejercicio de poder de mercado por los generadores, con relación al diseño de mercado separados. Johnsen et al. (2004) reportan evidencia empírica en el mercado eléctrico noruego, observando que los precios en los mercados locales son mayores durante los períodos de congestión en la transmisión, lo que posibilitó el ejercicio de poder de mercado. Bompard et al. (2006), aplicando la teoría de juegos, analizan el impacto de las restricciones de la red eléctrica en la performance de un mercado oligopólico; observando que un refuerzo en la transmisión puede mitigar el comportamiento anticompetitivo de los grandes generadores; sin embargo, solo estudia el caso de un mercado eléctrico con generación termoeléctrica.

10 ISO: Operador del Sistema Independiente. Sin fines de lucro, fuera del control de los agentes o pequeños grupos de interés del mercado. También opera el mercado de corto plazo. V. S. Stoft (2002) p. 447.

11 Pool: Mercado centralizado que usa compensaciones para pagar diferentes precios a diferentes ofertantes en el mismo momento y lugar. Stoft (2002) p. 224. 


\section{Jaime E. Luyo}

De la Cruz y García (2003) analizan la problemática de la actividad de transmisión en Perú, recomendando que para incentivar la expansión de los servicios de transmisión se requiere mejorar las señales de precios, entre las cuales están los costos de congestión asociados a los límites de capacidad de las líneas y que, previamente, se deben emular los potenciales riesgos de poder de mercado.

\section{EL SISTEMA DE TRANSMISIÓN ELÉCTRICO PERUANO}

El segmento de transmisión es considerado, a nivel internacional, como monopolio natural, debido a sus características físicas, que no hacen posible la competencia, y a la economía de escala ${ }^{12}$. Debido a las características del marco regulatorio existente, durante los últimos años en el Perú, el desarrollo de la transmisión ha sido muy limitado. El sistema actualmente existente adolece de serias deficiencias; y la seguridad del sistema, por este motivo, es causa de preocupación. Un análisis reciente de las condiciones de seguridad han puesto en evidencia que el sistema es bastante inseguro, en el sentido que la falla de algunas de sus líneas de transmisión podría causar apagones con una alta probabilidad.

Desde el punto de vista de la inversión, las reglas existentes de la Ley de Concesiones Eléctricas (Ley N. ${ }^{\circ} 25844,19-11-1992$, LCE) para la expansión de la transmisión no garantizan la recuperación de la inversión, dado que el método en base al sistema económicamente adaptado debe ser revisado cada cierto tiempo. La preocupación del COES, respecto a la seguridad del sistema, está centrada, actualmente, en la insuficiente capacidad de transmisión eléctrica en ciertas zonas del país -en particular, en las líneas que vinculan la región central con las regiones norte y sur del país-; y, por otro lado, la congestión en las líneas de transmisión pueden producir prácticas anti-competitivas ${ }^{13}$. El sistema de transmisión principal (a $220 \mathrm{KV}$ y $138 \mathrm{KV}$ ) tiene más de $3700 \mathrm{~km}$ de líneas que cubren la mayor parte del territorio nacional conformando el Sistema Eléctrico Interconectado Nacional (SEIN); notándose que la línea que une la región central con el Sur (Campo Armiño-Socabaya), y la que une la región central con el norte (Zapallal-Paramonga Nueva-Chimbote), están congestionadas ${ }^{14}$ periódicamente. Estas conexiones son radiales y más fáciles de analizar; por lo que en el presente trabajo, por su mayor

12 Ver Joskow, 2005; Hunt, 2002.

13 Ver Borenstein, Bushnell, Stoft, 2000.

14 COES. Comité de Operación Económica del Sistema, 2005. 


\section{Efectos de la congestión de redes de transmisión en mercados eléctricos}

complejidad, estudiaremos los efectos de la congestión de la red de transmisión de la región central del SEIN que contiene loops (flujos paralelos, y de ida y vuelta) y concentra el mayor nivel de demanda total nacional, para el análisis de PM.

Como expusimos en la presentación de la problemática del mercado eléctrico peruano (Luyo, 2006); la sequía del año 2004 evidenció problemas en el modelo de mercado, revelándose con la elevación excesiva de los precios de la electricidad en el mercado spot (transacciones inmediatas, corto plazo); observándose que el sistema eléctrico peruano, con una generación mayormente dependiente del recurso hidráulico, es vulnerable con relación a la incertidumbre del recurso hidráulico para la generación de electricidad y que, además, posibilita la utilización estratégica intertemporal de este recurso (Luyo, 2008).

Además, la Ley N. ${ }^{\circ} 28832$, del 23-07-2006, promulgada por el gobierno (Ley para la Generación Eficiente, LGE), tiende a desregular el mercado eléctrico al por mayor, reduciendo la intervención del Osinerg; lo que, considerando el alto índice de concentración horizontal en el mercado mayorista peruano (Herfindahl-Hirshmann Index $=2700$ ), podría incentivar las prácticas anticompetitivas por las empresas de generación dominantes. Esta ley, por otro lado, ante la falta de la necesaria independencia del COES de los agentes en una economía de mercado, como operador del mercado eléctrico (y del SEIN), contradictoriamente, ha reforzado la intervención de estos en su gobierno y ampliado sus funciones de interés público.

Por todo lo expuesto, y dada la importancia de la última medida (la LGE) orientada hacia una mayor desregulación del Mercado Eléctrico Mayorista Peruano (MeP); la investigación de los efectos de la congestión de la transmisión en la competencia adquiere plena actualidad, máxime si además el presidente de Osinerg (ahora Osinergmin), recomienda su estudio ${ }^{15}$, y el Gerente General de Luz del Sur expresa una preocupación similar ${ }^{16}$.

15 “...deben analizarse los potenciales problemas de poder de mercado...”. Dammert, 2005, p. 120.

16 "La ley define el sector de generación como un mercado de competencia, lo cual no es verdad, es un oligopolio...". El Comercio, 21 de octubre de 2006. 


\section{Jaime €. Luyo}

\section{MODELO TEÓRICO}

Luyo (2006) ha expuesto que el mercado eléctrico peruano (MeP) corresponde a un diseño pool de precios spot nodales, operado por un ISO (Operador Independiente del Sistema) que determina el equilibrio de mercado cada hora (o menos); además Luyo (2008) ha diagnosticado un comportamiento oligopólico en la generación. Con estas consideraciones, y dentro del espíritu de Borenstein et al. (2000), Hogan (1997), Harvey y Hogan (2000), Ehrenman y Neuhoff (2004), y Bompard et al. (2006), desarrollaremos el modelado del $\mathrm{MeP}$ para estudiar el efecto de la congestión de la transmisión en la competencia dentro del mercado de electricidad.

Los mercados de electricidad en la práctica tienden a comportarse estructuralmente como oligopolios; es decir, entre el monopolio y la competencia perfecta. Similarmente al comportamiento de otros mercados, los productores (generadores) pueden tender a buscar mayor beneficio, restringiendo la capacidad de producción para elevar los precios con relación a los niveles competitivos, lo que puede manifestarse como el ejercicio de poder de mercado.

En el trabajo (Luyo, 2008) se ha estudiado una de las formas de PM (a nivel global, sistémico) para sistemas de generación hidrotérmica, como en el mercado peruano; otra de las modalidades de PM más extendidas y que distingue al mercado eléctrico de otros, el Poder de Mercado Local, que es el que se posibilita cuando las líneas de transmisión eléctrica se congestionan, debiendo, sin embargo, mantenerse el balance instantáneo entre las potencias inyectadas por los generadores y las consumidas por las cargas en toda la red de transmisión que soporta el mercado eléctrico al por mayor, ya que se tienen que satisfacer las leyes físicas de Kirchhoff. En el presente trabajo, también se adopta el enfoque Nash-Cournot, y se considera la red eléctrica de interconexión del mercado, y no una línea aislada; lo que permitirá internalizar las externalidades debido a los loops (flujos paralelos, y de ida y vuelta) que se producen en una red eléctrica real; cuyo detalle de modelización se presenta en el anexo. 


\section{Efectos de la congestión de redes de transmisión en mercados eléctricos}

\section{CONCLUSIONES}

- Consideramos que en el anterior régimen de empresas integradas verticalmente (generación, transmisión, distribución y comercialización), el reforzamiento y expansión de la transmisión eléctrica se realizaba con el criterio de confiabilidad de ingeniería, considerando que existían monopolios por áreas geográficas y que erróneamente aún se mantiene; mientras que, después de 1990, el nuevo criterio es el de confiabilidad económica en un mercado mayorista de competencia en el segmento de generación, donde la configuración de la red de transmisión tendrá impacto en la posibilidad de ejercicio de poder de mercado por los productores (generadores).

- Tomando en cuenta la congestión en algunas líneas principales de transmisión eléctrica del SEIN; y que, además, actualmente se está produciendo la congestión del gasoducto (línea de gas) que abastece las centrales termoeléctricas, lo que condicionará el uso de unidades de generación diesel de propiedad de la empresa dominante (Endesa); es muy probable de que se produzca un incremento del precio de la electricidad en el corto plazo. Desde la crisis del 2004, se ha venido planteando la necesidad de que se diera la autorización oficial para que la empresa estatal ELECTROPERÚ instale una planta de generación termoeléctrica de 500MW, y así haya competencia en el mercado eléctrico peruano y, además, se tenga la suficiente reserva de generación para la seguridad del sistema.

- La congestión de una línea principal que conecta dos regiones del país, producirá la separación del mercado integrado existente antes de la congestión, en mercados aislados donde se posibilitará el ejercicio de poder de mercado por la empresa de generación dominante en cada uno de estos mercados. Esta situación se produjo en el caso de la congestión de la línea Zapallal-Paramonga Nueva-Chimbote, durante el año 2007 que se ha analizado en el presente artículo.

- La congestión en una línea de la red de transmisión eléctrica es suficiente para afectar los precios en todas las barras de la red (externalidad) con respecto al precio competitivo.

- Se ha presentado un marco analítico para el estudio de la congestión en la transmisión eléctrica y sus efectos en la competencia en el mercado eléctrico mayorista de generación. 


\section{Jaime E. Luyo}

- Ante la evidente ineficacia de la Ley de Generación Eficiente (LGE) que no ha asegurado la suficiencia de generación eléctrica, habiéndose declarado desiertas las últimas subastas de contratos y continuado el retiro de potencia y energía del SEIN por los distribuidores sin tener contratos vigentes; el Gobierno, para afrontar estos problemas, se ha visto obligado a dictar en los últimos años, diversas medidas paliativas modificando la LGE y la LCE, y los D.U. N. ${ }^{\text {os }}$ 007-2004, 035-2006; y 046-2007, del 25-11-2007, que modifica por decreto la determinación del nivel de los costos marginales del SEIN al haberse detectado congestión en las líneas de transmisión. Recientemente, el 03-01-2008, se promulgó la Ley N. ${ }^{\circ} 20179$, que legaliza los retiros de energía eléctrica sin contrato. Todos estos hechos son resultado de la disputa, atizada por la LGE, entre el regulador y las empresas de generación que hacen uso de su PM al no presentarse a las subastas (colusión tácita) para cubrir la demanda del servicio público de electricidad y preferir participar en el mercado spot, además de su renuencia a invertir en ampliación de la capacidad de generación y de transmisión del SEIN.

- Dada la actual gobernanza del COES y las disputas entre este, el regulador, y los agentes; a similitud de otros países, se debe institucionalizar un Comité de Monitoreo y Vigilancia del Mercado Eléctrico, con autonomía e independencia, e integrado por expertos.

- Finalmente, por lo expuesto, se puede anticipar que, ante el acentuado incremento de la máxima demanda en los años recientes (9.9\%, en 2008$)$, que era predecible ante el auge de la actividad minera y el crecimiento sostenido del PBI, y debido a la ausencia de un planeamiento estratégico de los recursos energéticos y políticas acertadas del uso; el país experimentará, probablemente, en el corto plazo un racionamiento eléctrico (o apagones programados), lo que repercutirá, sobre todo, en las nuevas inversiones y en los mejores deseos gubernamentales de mantener un crecimiento económico más competitivo que los países vecinos. 
Efectos de la congestión de redes de transmisión en mercados eléctricos

\section{ANEXO}

Figura N. ${ }^{\circ} 1$

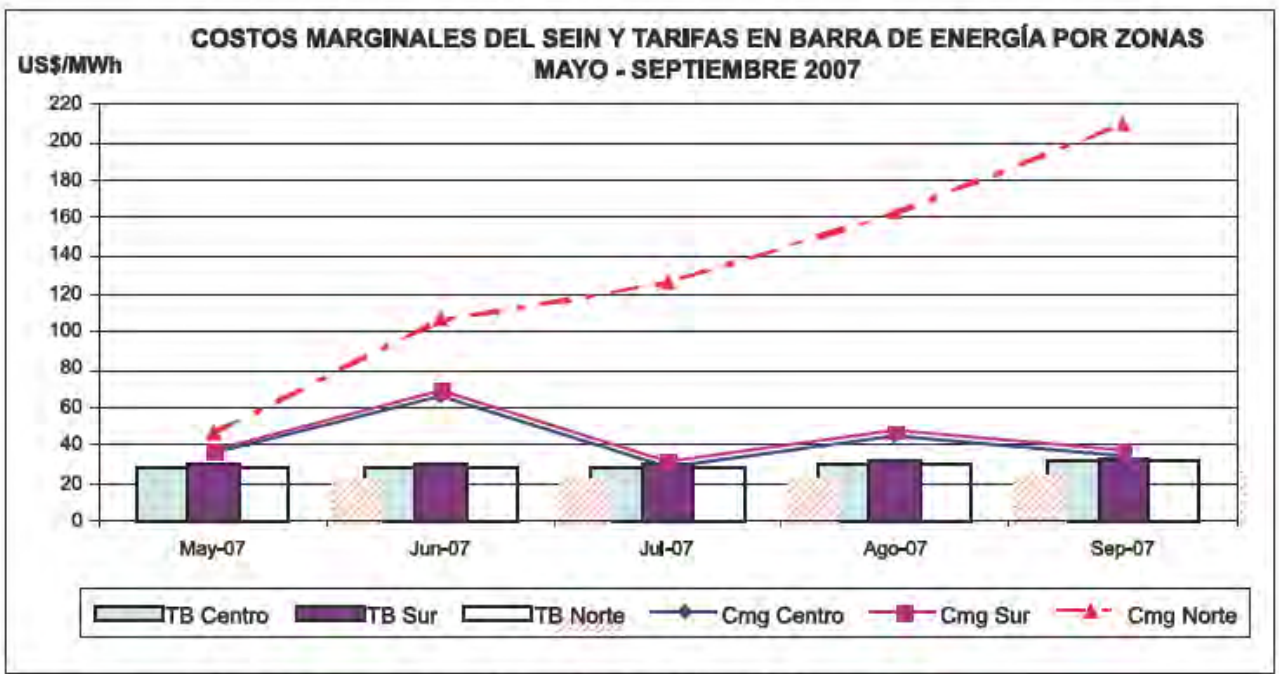

Nota: Las zonas Centro, Sur y Norte están referidas a les barras Santa Rosa, Socabaya y Trujilo respectivamente.

Fuente: ELECTROPERÚ S.A., Mesa Redonda. "Balance de los Procesos de Licitación para suministro de la Generación Eléctrica". Colegio de Ingenieros del Perú. Lima, 1 de febrero de 2008.

\section{COMPETENCIA EN UN MERCADO OLIGOPÓLICO CON PRODUCCIÓN HIDROTÉRMICA}

Cuando el mercado tiene producción hidrotérmica con almacenamiento hídrico, hay que tener en cuenta el flujo máximo de agua y volúmenes disponibles en los reservorios expresados en unidades de energía; y que, además, la producción $\left(p_{t}\right)$, así como la demanda, varían en el tiempo, donde el intercepto $\left(e_{t}\right)$ varía según el período. Se asume que en cada barra, excepto la barra de referencia, están acopladas un generador (o equivalente) hidroeléctrico y un termoeléctrico.

Las variables primales y duales variarán con el tiempo. El valor marginal del agua $\sigma$ en el corto plazo se mantiene, aproximadamente, constante en el tiempo; además la 


\section{Jaime E. Luyo}

congestión de las líneas de transmisión generalmente tiene cortos períodos de duración ${ }^{17}$.

Para el caso de un mercado competitivo, el problema de optimización a cargo del operador del sistema (ISO) es el siguiente.

Máximo Beneficio del Sistema

$$
\max _{p_{\mathrm{H}}, p_{\mathrm{T}}, q_{\mathrm{t}}} S^{\mathrm{s}}=\sum_{\mathrm{t}=1}^{\mathrm{p}} \frac{1}{2} q_{\mathrm{t}}^{\top} \cdot \mathrm{H} \cdot \mathrm{q}_{\mathrm{t}}+\mathrm{q}_{\mathrm{t}}^{\top} \mathrm{e}_{\mathrm{t}}-\left(\frac{1}{2} \mathrm{p}_{\mathrm{T} t}^{\top} \cdot \mathrm{B} \cdot \mathrm{p}_{\mathrm{T} t}+\mathrm{p}_{\mathrm{T} t \mathrm{t}}^{\top} \mathrm{a}\right)
$$

s.a. $\quad I_{d} \cdot q_{t}-I_{g} \cdot p_{t}=0 \quad, \forall t$

$$
\begin{array}{ll}
J\left(p_{t}-q_{t}\right) \leq f^{\max } & , \forall t \\
J\left(p_{t}-q_{t}\right) \geq-f^{\max } & , \forall t \\
p_{T t} \leq p_{T}^{\max } & , \forall t \\
p_{H t} \leq p_{H}^{\max } & \\
\sum_{t=1}^{p} p_{H t}=p_{H}^{\text {tot }} &
\end{array}
$$

además, $p_{t}=p_{H t}+p_{T t} \quad, \forall t, t=1, \ldots, P, P$ : número de periodos

también, $p_{H t}, p_{T t}, q_{t} \geq 0$, ya que los productores compiten para maximizar su beneficio; y también en el lado de la demanda se maximiza la utilidad.

$p_{t}, q_{t}$ : Vectores Gx1 y Dx1, de generación, y de demanda; $\mathrm{G}$ es el número de generadores, y $\mathrm{D}$ el número de cargas.

$f_{\max }: \quad$ Vector lx1 de los flujos límite en las "l" líneas de transmisión, MW.

17 Depende de la variación en el tiempo, del nivel de la demanda del mercado, que también se representa en el modelo. 


\section{Efectos de la congestión de redes de transmisión en mercados eléctricos}

$\mathrm{p}_{\mathrm{H}}^{\text {tot }}$ : Capacidad del embalse.

$p_{H}^{\max }$ : Vector de capacidad máxima en los generadores hidráulicos, MW.

$\mathrm{p}_{\mathrm{T}}^{\min }$ : Vector de capacidad mínima en los generadores térmicos, MW.

$I_{g}, I_{d}$ : Vectores fila identidad, con un número de elementos igual a generadores y cargas

$J$ Matriz $l(n-1)$ de transferencia de red con $l$ líneas y $n$ barras

$J_{G}, J_{D}$ : Matriz de transferencia correspondiente a barras de generación, y barras de carga.

$\gamma_{\mathrm{Nt}}$ : Precio en el nodo de referencia.

$\lambda_{t}$ : Vector de precios nodales; $\lambda_{G t}$, en barras de generación; $\lambda_{D t}$, en barras de carga.

$\mu_{\mathrm{t}}^{+}, \mu_{\mathrm{t}}$ : Vectores de variables duales, de restricciones de flujo en las líneas en el sentido positivo y en el negativo.

$\omega_{\mathrm{Ht}}^{+}, \omega_{\mathrm{Tt}}$ : Vectores de variables duales, de restricciones de capacidad máximo y mínimo en los generadores hidráulicos y térmicos.

$p^{T}, \int_{G}^{T}$ : Vector y matriz transpuestos. T es operación de transposición de vectores o matrices.

$c_{g}\left(p_{g}\right)=a_{g}+b_{g}-p_{g}$ : Costo marginal del generador $g$

$a, e_{t}$ : Vectores columna con elementos $a_{g}, y e_{d t}$ correspondientes al costo marginal de producción, y a la demanda, donde $g=1, \ldots, \mathrm{G}, d=1, \ldots, \mathrm{D}$.

$v\left(q_{d t}\right)=e_{d t}+h_{d} \cdot q_{d t}$ : Función inversa de demanda

$B, H$ : Matrices diagonales con elementos $b_{g}, y h_{d}$, correspondientes a la producción y la demanda.

El lagrangiano para el sistema es:

$$
\begin{aligned}
\mathcal{L}= & S^{S}-\sum_{t}^{p}\left\{\gamma_{N}\left[I_{d} \cdot q_{t}-I_{g} \cdot\left(p_{H t}+p_{T t}\right)\right]+\mu_{t}^{+T}\left[J\left(p_{H t}+p_{T t}-q_{t}\right)-f\right]\right. \\
& \left.+\mu_{t}^{-T}\left[-f-J\left(p_{H t}+p_{T t}-q_{t}\right)\right]+\omega_{H t}^{T}\left(p_{H t}-p_{H}^{\max }\right)+\omega_{T t}^{+T}\left(p_{T t}-p_{T}^{\max }\right)\right\} \\
& -\sigma\left(\sum_{t}^{p} p_{H t}-p_{H}^{\text {tot }}\right)
\end{aligned}
$$




\section{Jaime €. Luyo}

aplicando las condiciones de KKT

$$
\begin{aligned}
& \frac{\partial \mathcal{L}}{\partial \mathrm{p}_{\mathrm{Ht}}}=\gamma_{\mathrm{Nt}} \mathrm{I}_{\mathrm{g}}^{\mathrm{T}}-\mathrm{J}_{\mathrm{G}}^{\mathrm{T}} \cdot\left(\mu_{\mathrm{t}}^{+}-\mu_{\mathrm{t}}^{-}\right)-\omega_{\mathrm{Ht}}-\sigma=0 \\
& \lambda_{G t}=\gamma_{N t} I_{g}^{T}-J_{G}^{T} \cdot\left(\mu_{t}^{+}-\mu_{t}^{-}\right)=\omega_{H_{t}}+\sigma
\end{aligned}
$$

(2)

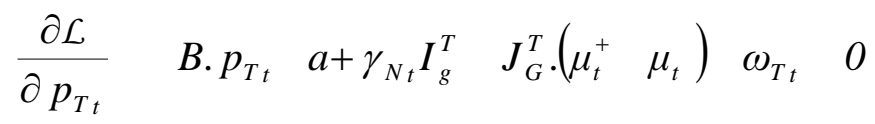

$$
\lambda_{\mathrm{Gt}}=\gamma_{\mathrm{Nt}} \mathbf{I}_{\mathrm{g}}^{\mathrm{T}} \quad \mathrm{J}_{\mathrm{G}}^{\mathrm{T}} \cdot\left(\mu_{\mathrm{t}}^{+} \quad \mu_{\mathrm{t}}\right)=\mathrm{B} \cdot \mathrm{p}_{\mathrm{Tt}}+\mathrm{a}+\omega_{\mathrm{Tt}}
$$$$
p_{T t} \quad(B)^{1}\left[\begin{array}{lll}
\lambda_{G t} & \omega_{T t} & a
\end{array}\right]
$$

$$
\frac{\partial \mathcal{L}}{\partial \mathrm{q}} \quad \text { H. } \mathrm{q}_{\mathrm{t}}+\mathrm{e}_{\mathrm{t}} \gamma_{\mathrm{Nt}} \mathrm{I}_{\mathrm{d}}^{\mathrm{T}}+\mathrm{J}_{\mathrm{D}}^{\mathrm{T}}\left(\begin{array}{ll}
\mu_{\mathrm{t}}^{+} & \mu_{\mathrm{t}}
\end{array}\right) \quad 0
$$

$$
\lambda_{\mathrm{Dt}}=\gamma_{\mathrm{N} t} \mathrm{I}_{\mathrm{D}}^{\mathrm{T}}-\mathrm{J}_{\mathrm{D}}^{\mathrm{T}} \cdot\left(\mu_{\mathrm{t}}^{+}-\mu_{\mathrm{t}}^{-}\right)=\mathrm{H} \cdot \mathrm{q}_{\mathrm{t}}+\mathrm{e}_{\mathrm{t}}
$$

donde $\omega_{\mathrm{Ht}}$ y $\omega_{\mathrm{Tt}}$, son la renta de escasez de capacidad hidroeléctrica y la renta de escasez de capacidad termoeléctrica, en cada periodo

Cuando la competencia es oligopólica, según el enfoque de Cournot, el máximo beneficio de cada generador hidrotérmico se puede expresar como:

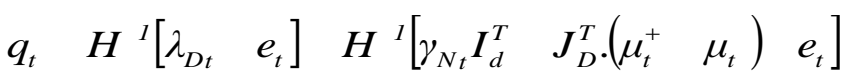

s.a. $\quad J\left(p_{t}-q_{t}\right) \leq f^{\max } \quad, \forall t$ 


$$
\begin{array}{ll}
J\left(p_{t}-q_{t}\right) \geq-f^{\max } & , \forall t \\
p_{T t} \leq p_{T}^{\max } & , \forall t \\
p_{H t} \leq p_{H}^{\max } & , \forall t \\
\sum_{t=1}^{p} p_{H t}=p_{H}^{\text {tot }} &
\end{array}
$$

además, $p_{t}=p_{H t}+p_{T t}, \forall t, \quad p_{H t}, p_{T t}, q_{t}>0$

De (6) se halla que

$$
\lambda_{\mathrm{Gt}}=\mathrm{a}+B \cdot \mathrm{p}_{\mathrm{Tt}}+\mathrm{J}_{\mathrm{G}}^{\mathrm{T}} \cdot\left(\mu_{\mathrm{t}}^{+}-\mu_{\mathrm{t}}\right)-\frac{\mathrm{p}_{\mathrm{t}}}{\mathrm{I}_{\mathrm{d}} \cdot \mathrm{H}^{1} \mathrm{I}_{\mathrm{d}}^{\mathrm{T}}}+\omega_{\mathrm{Tt}}
$$

y además, de (2) se tiene que

$$
\lambda_{\mathrm{g}_{\mathrm{t}}}=\gamma_{\mathrm{Nt}}-\mathrm{J}_{\mathrm{g}}^{\mathrm{T}} \cdot\left(\mu_{\mathrm{t}}^{+}-\mu_{\mathrm{t}}^{-}\right)
$$

donde, $\int_{g}^{\top}$ es el vector fila de $J$ correspondiente al generador $g$ en el ordenamiento de $\lambda_{\mathrm{Gt}}(3)$.

La función lagrangiana resulta

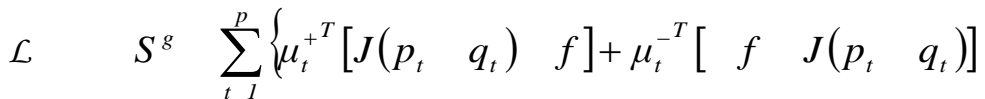

$$
\begin{aligned}
& \left.+\omega_{H t}\left(\begin{array}{ll}
\mathrm{p}_{H \mathrm{t}} & \mathrm{p}_{\mathrm{H}}^{\max }
\end{array}\right)+\omega_{\mathrm{Tt}}\left(\begin{array}{ll}
\mathrm{p}_{\mathrm{T} t} & \mathrm{p}_{\mathrm{T}}^{\max }
\end{array}\right)\right\} \quad \sigma\left(\begin{array}{ll}
\sum_{\mathrm{t} l 1}^{\mathrm{p}} \mathrm{p}_{H \mathrm{t}} & \mathrm{p}_{H}^{\text {tot }}
\end{array}\right)
\end{aligned}
$$

considerando (8) y (9) en (7) y aplicando las condiciones KKT 


\section{Jaime €. Luyo}

$$
\begin{aligned}
& \frac{\partial \mathcal{L}}{\partial p_{H t}} \quad \lambda_{g_{t}}+\frac{p_{g_{t}}}{I_{d} \cdot H^{-1} \cdot I_{d}^{\top}} \quad J_{g}^{\top} \cdot\left(\begin{array}{llllll}
\mu_{t}^{+} & \mu_{t}^{-}
\end{array}\right) \omega_{H t} \quad \sigma \quad 0 \\
& \frac{\partial \mathcal{L}}{\partial p_{T t}} \quad \lambda_{g_{t}}+\frac{p_{g_{t}}}{I_{d} H^{-1} \cdot I_{d}^{T}} \quad J_{g}^{T} \cdot\left({ }^{+} \mu_{t}^{-}\right) \omega_{T t} \quad \text { b. } p_{T t} \quad a \quad 0
\end{aligned}
$$

además: $\lambda_{\mathrm{gH}_{\mathrm{t}}} \quad \lambda_{\mathrm{gT}_{\mathrm{t}}} \quad \lambda_{\mathrm{g}_{\mathrm{t}}}$

y el precio en la barra de generación resulta, en forma matricial

$$
\begin{aligned}
& \text { (10) } \lambda_{\mathrm{Gt}}=\sigma+\mathrm{J}_{\mathrm{G}}^{\mathrm{T}} \cdot\left(\begin{array}{ll}
\mu_{\mathrm{t}}^{+} & \mu_{\mathrm{t}}
\end{array}\right) \frac{\mathrm{P}_{\mathrm{t}}}{\mathrm{I}_{\mathrm{d}} \cdot \mathrm{H}^{1} \cdot \mathrm{I}_{\mathrm{d}}^{\mathrm{T}}}+\omega_{\mathrm{Ht}} \\
& \text { (10a) } \lambda_{\mathrm{Gt}}=\mathrm{a}+B \cdot \mathrm{P}_{\mathrm{Tt}}+\mathrm{J}_{\mathrm{G}}^{\mathrm{T}} \cdot\left(\mu_{\mathrm{t}}^{+}-\mu_{\mathrm{t}}\right)-\frac{\mathrm{P}_{\mathrm{t}}}{\mathrm{I}_{\mathrm{d}} \cdot \mathrm{H}^{1} \cdot \mathrm{I}_{\mathrm{d}}^{\mathrm{T}}}+\omega_{\mathrm{Tt}}
\end{aligned}
$$

Se puede observar de (2) y (3) correspondien e a un mercado competitivo con restricciones en la transmisión y la expresión (10) de competencia oligopólica se produce un incremento de los precios a través del manejo estratégico de la producción $\left(\frac{P_{t}}{I_{d} \cdot H^{1} \cdot I_{d}^{\top}}\right.$ en (10)) y, además, el efecto de congestión en las líneas $\left(J_{G}^{\top} \cdot\left(\mu_{t}^{+}-\mu_{t}^{-}\right)\right)$ que representa la renta de congestión

Para el caso competitivo sin congestión en la transmisión, se tiene que

$$
\gamma_{\mathrm{Nt}} \mathrm{I}_{\mathrm{g}}^{\top}=\sigma+\omega_{\mathrm{Ht}} \quad, \quad \forall \mathrm{t}
$$

el precio es igual en todos los nodos de la red eléctrica. Y si, además no se produce limitación de capacidad en todos los generadores, el valor marginal del agua se iguala a un nivel equivalente en todos los embalses por un efecto de arbitraje; lo que significa, además, una regulación automática a través del tiempo, debido a la participación de la producción hidroeléctrica con embalses.

Las restricciones de complementariedad para el modelo de Cournot desarrollado, 


\section{Efectos de la congestión de redes de transmisión en mercados eléctricos}

(11)

\begin{tabular}{|c|c|c|c|c|}
\hline$<\mu_{\mathrm{t}}^{+}$ & , & $J\left(p_{t}\right.$ & $\left.q_{t}\right) \quad f^{\max }>$ & 0 \\
\hline$\mu_{\mathrm{t}}$ & , & $J\left(p_{t}\right.$ & $\left.q_{t}\right)+f^{\max }>$ & 0 \\
\hline$\omega_{H_{t}}$ & , & $\mathrm{p}_{\mathrm{H} t}$ & $\mathrm{p}_{\mathrm{H}}^{\max }>$ & $=0$ \\
\hline & , & $\mathrm{p}_{\mathrm{Tt}}$ & $\mathrm{p}_{\mathrm{T}}^{\max }>$ & $=0$ \\
\hline
\end{tabular}

y la restricción

$$
\begin{aligned}
& \sum_{t=1}^{p} p_{H t}-p_{H}^{\text {tot }}=0 \\
& p_{H t}, p_{T t}, q_{t} \geq 0, \forall t
\end{aligned}
$$

que con las relaciones (10) igualadas a cero y (9), conforman un MCP no-lineal.

$\mu_{\mathrm{t}}^{+}, \mu_{\mathrm{t}}^{-}$: representan las rentas marginales de congestión, y las $\omega_{\mathrm{Ht}}, \omega_{T_{\mathrm{t}}}$ las rentas marginales de escasez de capacidad

Las líneas de transmisión pueden tene tre estados descongestión, congestión en la dirección positiva y congestión en la dirección negativa También, los generadores pueden tener tres estados sin limitación de capacidad con límite superior y con límite inferior Entonces, si se tienen $L$ líneas que pueden estar congestionadas (con $3^{L}$ estados posibles), y $G$ generadores, que pueden estar con límite de capacidad (con $3^{G}$ estados posibles); cada generador en competencia tendría que considerar $3^{L} .3^{G}$ alternativas, lo cual sería excesivo e impráctico conforme aumenta el número de elementos en la red.

Las alternativas se pueden reducir, al considerar que, para el problema de maximización del beneficio de un generador, el límite inferior puede ser ignorado, ya que produciría un beneficio nulo; además, para alcanzar el máximo beneficio o ganancia, según Bompard et al. (2006a), implica que los generadores no tengan limitación de capacidad lo que da como resultado que las alternativas se reduzcan a $3^{L}$.

Adicionalmente, si consideramos la operación real de la red de transmisión eléctrica, en un determinado período de funcionamiento, sólo se podrá producir en un solo estado. El estado de una determinada línea puede ser, entonces, preespecificado a 


\section{Jaime $\in$. Luyo}

través de una simulación o a través de los registros históricos de operaciones del sistema eléctrico. Esto significa que los multiplicadores de Lagrange $\mu_{\mathrm{t}}, \mu_{\mathrm{t}}^{-}$serán nulos cuando una determinada línea está descongestionada; y $\mu_{\mathrm{t}}^{+} \neq 0\left(\mu_{\mathrm{t}}^{-}=0\right)$, cuando el flujo de potencia en la dirección positiva está limitado; y $\mu_{\mathrm{t}}^{-} \neq 0\left(\mu_{\mathrm{t}}^{+}=0\right)$, cuando hay congestión del flujo en la dirección negativa; lo que, en consecuencia, permite linealizar las restricciones de complementariedad.

Siendo el objetivo investigar los efectos de la congestión de las líneas de transmisión en la competencia; si tomamos en cuenta que el balance oferta-demanda en el mercado se debe mantener siempre, y que en todo análisis de flujo de potencia en la transmisión la producción está dada, y que los flujos se redistribuyen en la red lley nodal de Kirchhoff); para fines de simulación, se puede considerar, entonces, que la producción $q_{t}$ es el resultado de resolver el equilibrio Cournot-Nash sin congestión del juego multiperiodo. Además, la congestión tiene un comportamiento estático, es decir, está vinculada solo a un determinado periodo $t$ de operación del mercado ${ }^{18}$ (que corresponde al juego estático (simultáneo) entre empresas en dicho periodo).

\section{CASO DE LA CONGESTIÓN EN LA LÍNEA LIMA-CHIMBOTE 220KV}

Este caso corresponde la situación reportada en la Figura 1, y que puede representarse en una forma sencilla mediante el gráfico siguiente:
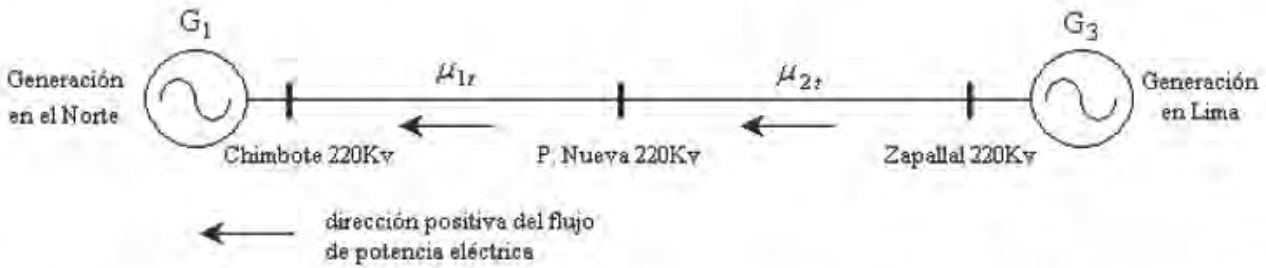

Asumiendo que la barra 2 es la barra de referencia, y aplicando la metodología diseñada en (Schweppe, 1988), se obtiene que la matriz de transferencia de red resulta:

$$
J=\left[\begin{array}{cc}
-1 & 0 \\
0 & 1
\end{array}\right]
$$

18 El flujo de potencia en la líneas de transmisión eléctrica tiene comportamiento estático y no dinámico. Este se distribuye (o redistribuye) según la magnitud de la impedancia eléctrica de las líneas y del nivel de produccí́n de los generadores. Ver Saadat (2002). 


\section{efectos de la congestión de redes de transmisión en mercados eléctricos}

Considerando la generación termoeléctrica (ecuación (10a)), y que la dirección del flujo de electricidad es positiva; resulta que el precio de la energía eléctrica en la barra 1 correspondiente a demanda de la zona norte del país es:

$$
\lambda_{G 1 t}=a_{1}+b_{1} p_{T 1 t}+\mu_{1 t}-\frac{p_{1 t}}{1 / h_{1}+1 / h_{3}}+\omega_{T 1 t}
$$

y como $\mu_{1 \mathrm{t}}=\mu_{2 \mathrm{t}}$ que es la misma línea; en la zona central de Lima, el precio resulta

$$
\lambda_{G 3 t}=a_{3}+b_{3} p_{T 3 t}+\mu_{3 t}-\frac{p_{3 t}}{1 / h_{1}+1 / h_{3}}+\omega_{T 3 t}
$$

se produce una renta de congestión $\left(+\mu_{1 t}\right)$ que aumenta el precio en la zona norte. Si se considera que no hay renta de escasez de capacidad termoeléctrica $\left(\omega_{T 1 \mathrm{t}}=\omega_{\mathrm{T} 2 \mathrm{t}}=0\right)$ y que tampoco se produce en este caso (una sola línea) el efecto oligopólico, al descomponerse el mercado integrado (periodo pre-congestión) en dos mercados aislados y no hay competencia entre el generador equivalente G1 y G3; se produce, entonces, un escenario que, debido a la congestión, ya no es posible que la producción de la zona centro (Lima), pueda atender una mayor demanda de la zona norte; lo cual posibilita que se produzca una estrategia de withholding por parte de un generador térmico retirando de producción unidades eficientes y reemplazándolas por otras ineficientes y más caras aumentando su poder de mercado local, y aumentando sus beneficios. Esto se puede mostrar gráficamente (ver Figura 2).






\section{Jaime $\in$. Luyo}

El intercepto A pasa a A', lo que equivale a un incremento de la magnitud del parámetro $a_{1}$.

Esto explica, en parte, el incremento excesivo de los costos marginales reportado en el gráfico de la Figura 1, ya que las centrales térmicas que cubrían el pico de la demanda (en periodo de máxima demanda) eran de combustible Diesel, que es muy caro, y que determinaron los costos marginales en la zona norte del país para el periodo representado; lo cual afectaría negativamente al resto del país si se consideraba, erróneamente, como un solo mercado y competitivo. Una situación similar se puede producir en la zona centro si se tiene un generador dominante

\section{CASO: CONGESTIÓN DE UNA LÍNEA DE UNA RED DE TRANSMISIÓN}

Considerando el término $\mathrm{J}_{\mathrm{G}}^{\mathrm{T}} \cdot\left(\mu_{\mathrm{t}}^{+}-\mu_{\mathrm{t}}^{-}\right)$de la ecuación (10a); asumiendo que los flujos son positivos en la red de transmisión de electricidad y que solo una línea i está congestionada $\mu_{\mathrm{it}}$; resulta:

$$
\mathrm{J}_{\mathrm{G}}^{\mathrm{T}} \cdot \mu_{\mathrm{t}}^{+}\left[\begin{array}{cccc}
\mathrm{J}_{11} & \mathrm{~J}_{12} & \cdots & \mathrm{J}_{\mathrm{In}} \\
\cdots & \cdots & \cdots & \cdots \\
\mathrm{J}_{\mathrm{i} 1} & \mathrm{~J}_{\mathrm{i} 2} & \cdots & \mathrm{J}_{\mathrm{in}} \\
\cdots & \cdots & \cdots & \cdots \\
\mathrm{J}_{\mathrm{G} 1} & \mathrm{~J}_{\mathrm{G} 2} & \cdots & \mathrm{J}_{\mathrm{Gn}}
\end{array}\right]\left[\begin{array}{c}
0 \\
\vdots \\
\mu_{\mathrm{it}} \\
\vdots \\
0
\end{array}\right]\left[\begin{array}{c}
\mathrm{J}_{\mathrm{i} 1} \\
\mathrm{~J}_{\mathrm{i} 2} \\
\vdots \\
\vdots \\
\mathrm{J}_{\mathrm{in}}
\end{array}\right] \mu_{\mathrm{it}}
$$

lo que expresa que basta que una sola línea esté congestionada para afectar los precios de la electricidad en todas las barras de la red de transmisión eléctrica. 


\section{Efectos de la congestión de redes de transmisión en mercados eléctricos}

\section{BIBLIOGRAFÍA}

Bompard, Ettore, MA Yuchao and Ragazzi, Elena (2006). "Microeconomic Analysis of the Physical Constrained Markets: Game Theory Application to Competitive Electricity markets", Higher Education and Research on Mobility Regulation and the Economics of Local Services, HERMES, Fundazione Collegio Carlos Alberto, Turin, Italy.

Borenstein, S.; Bushnell, James; Stoft, Steven (2000). The competitive effects of transmission capacity in a deregulated electricity industry, October 98, Revised October 1999, Revised version published in Rand Journal of Economics, Summer 2000.

Cho In Koo and Hyonsook Kim (2002). Market power and network constraint in a deregulated electricity market, Power Conference of the University of California Energy Institute, USA, December 12.

De La Cruz Sandoval, Ricardo; García Carpio, Raúl (2002). Mecanismos de competencia en generación de energía y su impacto en la eficiencia: El caso peruano. Consorcio de Investigación Económico y Social, CIES, febrero.

Decreto Ley 25844 "Ley de Concesiones Eléctricas”, 1992.

Ehrenmann, Andreas; Nuhoff, Karsten (2004). A comparison of electricity market design in networks. University of Cambridge, Department of Applied Economics, GWPE 0341, October.

Green, Richard (1998). Electricity Transmission Pricing: How much does it cost to get it wrong?, University of California Energy Institute, USA, PWP-058, april.

Harvey, Scott; Hogan, William (2000). Nodal and Zonal Congestion Management and the Exercise of Market Power, Harvard Electricity Policy Group and the Harvard-Japan Proyect on Energy and the Enviroment, USA, January.

Hogan, W. (1997). "A Market Power Model with Strategic Interaction in Electricity Networks", Harvard Institute for International Development, International Program on Privatization and Regulatory Reform, July.

Johnsen, Tor Amt; Kant, Shashi; Wolfram, Catherine (2004). Zonal Pricing and DemandSide Responsiveness in the Norwegian Electricity Market, Harvard Electricity Policy GroupUniversity of California Energy Institute, June1999, C. Convertion, November 2004. 


\section{Jaime $\in$. Luyo}

Joskow, Paul (2003). The Difficult Transition to Competitive Electricity Markets in the U.S., MIT, May.

Luyo, Jaime E. (2008). "Crisis de las reformas, poder de mercado y mitigación en el mercado eléctrico peruano". En Revista IECOS - UNI N. ${ }^{\circ}$ 5, año 3, Vol. I, Lima, Febrero 2008.

Luyo, Jaime E. (2008). Poder de Mercado y Mitigación en Sistemas de Generación Hidrotérmica. Caso del Mercado Eléctrico Peruano", ECI 2008, Encuentro Científico Internacional 2008, CONCYTEC, Lima, 2-4 enero 2008, www.eciperu.org.pe

Luyo, Jaime E. (2006). "Reforma del sector eléctrico en Perú: La crisis del 2004 y cambios necesarios", Revista de la Facultad de Ciencias Económicas, UNMSM, en edición septiembre/octubre 2006.

Saadat, Hadi (2002). Power System Analysis, Second edition. Boston, USA: McGrawHill.

Schweppe, F.C., Caramanis, M., Tabors, P., and Bohn, Roger E. (1988). Spot Pricing of Electricity, Kluwer Academic Publishers,

Stoft, Steven(1997). The Effect of the Transmission Grid on Market Power, paper \# LBNL-40479, E:O: Lawrence Berkeley National Laboratory, U. California, Berkeley, May 1997.

Twoney, P.; Green, Richar; Neuhoff; Karsten and Newbery, David (2005). "A Review of the Monitoring of Market Power: The Possible Roles of TSOs in Monitoring for Market Power Issues in Congested Transmission System", 05-02 Working Paper, Center for Energy and Environmental Policy Research, Department of Economics, Massachusetts Institute of Technology, MIT, USA, March.

Wolak, Frank A. (2003a). "Designing Competitive Wholesale Electricity Markets for Latin American Countries", Department of Economics, Stanford University, March 29.

Wu, Felix; Variya, Pravin, Spiller, Pablo and Oren, Shmuel (1996). "Folk Theorems on Transmission Access: Proofs and Counterexamples", Journal of Regulatory Economics, 10:5-23. 